Atrkivoc

Free to Authors and Readers
A Platinum Open Access Journal for Organic Chemistry
Paper

Arkivoc 2021, part iii, 197-209

\title{
Synthesis of novel isoxazoline and isoxazolidine derivatives: carboxylic acids and delta bicyclic lactones via the nucleophilic addition of bis(trimethylsilyl)ketene acetals to isoxazoles
}

Saulo C. Rosales-Amezcua, ${ }^{\text {a }}$ Ricardo Ballinas-Indili, ${ }^{a}$ Morelia E. López-Reyes, ${ }^{b}$ Héctor A. Rosas-Castañeda, ${ }^{a}$ R. Alfredo Toscano, ${ }^{\text {a }}$ Cecilio Álvarez-Toledano*a

aInstituto de Química, Universidad Nacional Autónoma de México (UNAM), Circuito Exterior s/n, Ciudad Universitaria, Cd. de México, 04510, México

${ }^{b}$ Departamento de Química, Universidad de Guadalajara, Blvd. Marcelino García Barragán 1421, Guadalajara, 44430, México

Email: cecilio@unam.mx

This paper is dedicated to the successful career of Professor Lanny Liebeskind

Received 02-25-21

Accepted 04-19-2021

Published on line $04-30-2021$

\section{Abstract}

Bis(trimethylsilyl)ketene acetals readily react with activated $N$-triflyl isoxazoles to selectively afford novel isoxazoline or isoxazolidine derivatives. The regioselectivity of the reaction strongly depends on the substrate substituents. When the isoxazole is substituted at the 5-position by a methyl or phenyl group, the lactonization product, i.e., the isoxazolidine derivative, is formed as a result of double nucleophilic addition of the ketene acetal. When the isoxazole is not substituted, the main product is the corresponding carboxylic acid, i.e., the isoxazoline derivative.

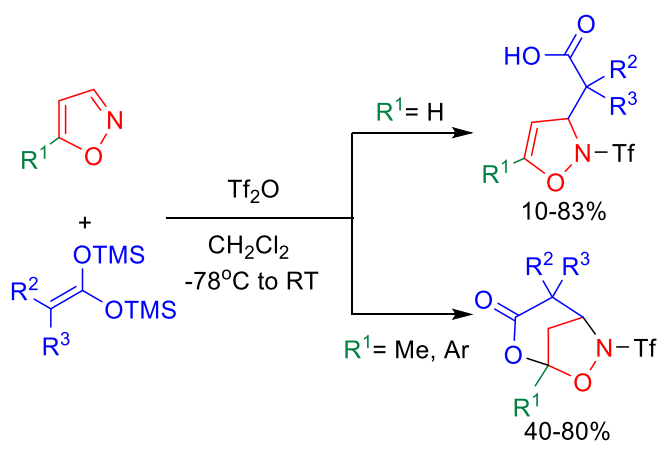

Keywords: Ketene acetals, isooxazoline, isoxazolidine, lactone, N-triflyl activation 


\section{Introduction}

Isoxazoline ${ }^{1-6}$ and isoxazolidine ${ }^{7-11}$ compounds represent an important class of five-membered heterocycles with contiguous nitrogen and oxygen atoms which have attracted significant attention, mainly due to their broad range of biological activities and potential application in the pharmaceutical industry. They are considered as privileged scaffolds in drug design and synthesis. ${ }^{7,12-18}$ Furthermore, these structures are found in a number of natural products. ${ }^{1,19-21}$

Over the last two decades, our research group and others have been interested in the nucleophilic addition of bis(trimethylsilyl)ketene acetals to different activated heterocyclic substrates ${ }^{22}$ such as pyridine, ${ }^{23-28}$ pyrazine, ${ }^{29-31}$ quinoline $^{32,33}$ imidazole, $^{34} 4$-azabenzimidazole ${ }^{35}$ and 4,5 -dihydrooxazole ${ }^{36}$ derivatives. In most cases, the main products of these reactions are carboxylic acids which can be transformed into the corresponding $\gamma$ or $\delta$ lactones via the addition of electrophilic species which promote an intramolecular cyclization. Only in a few cases, depending on the nature of the heterocycle and the activating agent, did the nucleophilic addition of the bis(trimethylsilyl)ketene acetal directly afford the lactonization product. ${ }^{23,28,30,31,36}$ The interest in these types of carboxylic acid and lactones lies in their potential biological properties, and their use as scaffolds in the synthesis of pharmaceutically interesting compounds. ${ }^{37-39}$

Among the examined substrates for the nucleophilic addition of bis(trimethylsilyl)ketene acetals, studies with isoxazole derivatives remain elusive. We envisaged the isoxazole structure as a good candidate to build 4isoxazoline and isoxazolidine derivatives with potential biological properties.

Herein, we report a methodology to selectively obtain novel 4-isoxazoline carboxylic acid or isoxazolidine$\delta$-bicyclic lactone derivatives via the nucleophilic addition of bis(trimethylsilyl)ketene acetals to isoxazoles. To promote the reaction, we decided to activate the substrate by introducing the strongly electron-withdrawing triflyl group at the 2-position of isoxazole. Additionally, the $\mathrm{CF}_{3} \mathrm{SO}_{2}$ group could confer potential biological activity to the product. ${ }^{40,41}$

\section{Results and Discussion}

We started our study with the activation of isoxazole 1 with triflic anhydride (1.2 equiv), followed by the addition of bis(trimethylsilyl)ketene acetal 2 at $-78^{\circ} \mathrm{C}$ in $\mathrm{CH}_{2} \mathrm{Cl}_{2}$ as the solvent (Scheme 1).

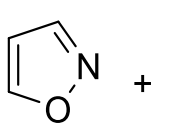

1

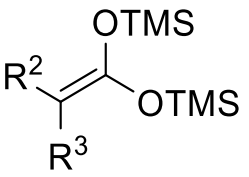

2

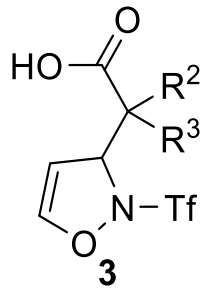

3

Scheme 1. Synthesis of 4-isoxazoline derivatives (3).

If substrate $\mathbf{1}$ is not activated with triflic anhydride, the carbon at the 3-position is not electrophilic enough and the reaction does not proceed (Table 1, entry 1 ).

As shown in Table 1, after triflic anhydride is added, the activation time has an important impact on the overall reaction effectiveness. When $\mathbf{1}$ was reacted with triflic anhydride for $1 \mathrm{~h}$, and then $\mathbf{2}$ is added and stirred 
for $24 \mathrm{~h}$, the 4 -isoxazoline carboxylic acid derivative $\mathbf{3}$ is obtained in $50 \%$ yield (Table 1, entry 2). However, if the reaction is activated for $3 \mathrm{~h}$, the yield increases to $81 \%$ (Table 1, entry 3). After $4 \mathrm{~h}$ activation, there is not a significant change in the yield (Table 1, entries 4 and 5).

Table 1. Activation times of isoxazole 1 and yields

\begin{tabular}{ccc}
\hline Entry & Activation time & Yield \% \\
\hline 1 & Without activating agent & N.R \\
2 & $1 \mathrm{~h}$ & 50 \\
3 & $3 \mathrm{~h}$ & 81 \\
4 & $4 \mathrm{~h}$ & 83 \\
5 & $6 \mathrm{~h}$ & 83 \\
\hline
\end{tabular}

Reagents and conditions: $\mathrm{Tf}_{2} \mathrm{O}$ (1.2 equiv), anhydrous $\mathrm{CH}_{2} \mathrm{Cl}_{2}(15 \mathrm{~mL}),-78^{\circ} \mathrm{C}$. N.R: not reaction.

In order to explore the scope of this reaction, we decided to examine the reaction of oxazole 1 with different bis(trimethylsilyl) ketene acetals 2 (Table 2). As expected, the nucleophilic addition reaction showed strong dependence on steric effects. When the bis(trimethylsilyl) ketene acetals are substituted by $R^{2}$ and $R^{3}$ substituents larger than methyl groups, i.e., cyclobutyl, cyclopentyl and cyclohexyl groups, the yields decreased dramatically as the steric-hindrance effects increased (Table 2). This result can be attributed to the presence of the voluminous $-\mathrm{SO}_{2} \mathrm{CF}_{3}$ group at the 2-position of the isoxazole, increasing the steric strain in the compound which led to product degradation.

Table 2. Yields of carboxylic acids $\mathbf{3}$ with different bis(trimethylsilyl) ketene acetals $\mathbf{2}$

\begin{tabular}{cccccc}
\hline Entry & $\mathrm{R}^{2}$ & & $\mathrm{R}^{3}$ & Product & Yield (\%) \\
\hline 1 & $\mathrm{CH}_{3}$ & & $\mathrm{CH}_{3}$ & $\mathbf{3 a}$ & 83 \\
2 & & $-\left(\mathrm{CH}_{2}\right)_{3^{-}}$ & & $3 \mathbf{3}$ & 41 \\
3 & & $-\left(\mathrm{CH}_{2}\right)_{4^{-}}$ & & $3 \mathbf{3}$ & 38 \\
4 & & $-\left(\mathrm{CH}_{2}\right)_{5^{-}}$ & & 3d & 10 \\
\hline
\end{tabular}

Conditions: Activation time $4 \mathrm{~h}, 1.2$ eq of 2 , overall reaction time $24 \mathrm{~h}$.

Compounds 3a-d were fully characterized using spectroscopic techniques. As a representative example, for compound $3 \mathrm{a}$, the presence of the carboxylic acid was clearly observed in the IR spectrum by two absorption bands: a broad band between 2800 and $3200 \mathrm{~cm}^{-1}(\mathrm{v}(\mathrm{OH}))$, and a sharp band at $1771 \mathrm{~cm}^{-1}(\mathrm{v}(\mathrm{C}=\mathrm{O}))$. The ${ }^{1} \mathrm{H}-\mathrm{NMR}$ spectrum showed a broad singlet at $10.54 \mathrm{ppm}$ corresponding to the hydrogen of the - $\mathrm{COOH}$ group, and a signal at $5.23 \mathrm{ppm}(\mathrm{m}, 1 \mathrm{H})$ corresponding to the proton at the 3-position of the isoxazoline (the site of the nucleophilic addition by the bis(TMS)ketene acetal). In the ${ }^{13} \mathrm{C}-\mathrm{NMR}$ spectrum, a signal at $181.3 \mathrm{ppm}$ is observed for the carboxylic carbon, and introduction of the triflyl group is confirmed by a quartet signal at $121.74 \mathrm{ppm}\left(J_{\mathrm{CF}} 323.2\right.$ $\mathrm{Hz}$ ). Moreover, the molecular structure of compound 3c was unambiguously confirmed by X-ray diffraction analysis (Figure 1). The molecular structure shows the carboxylic acid in an anti-conformation with respect to the triflyl group. 


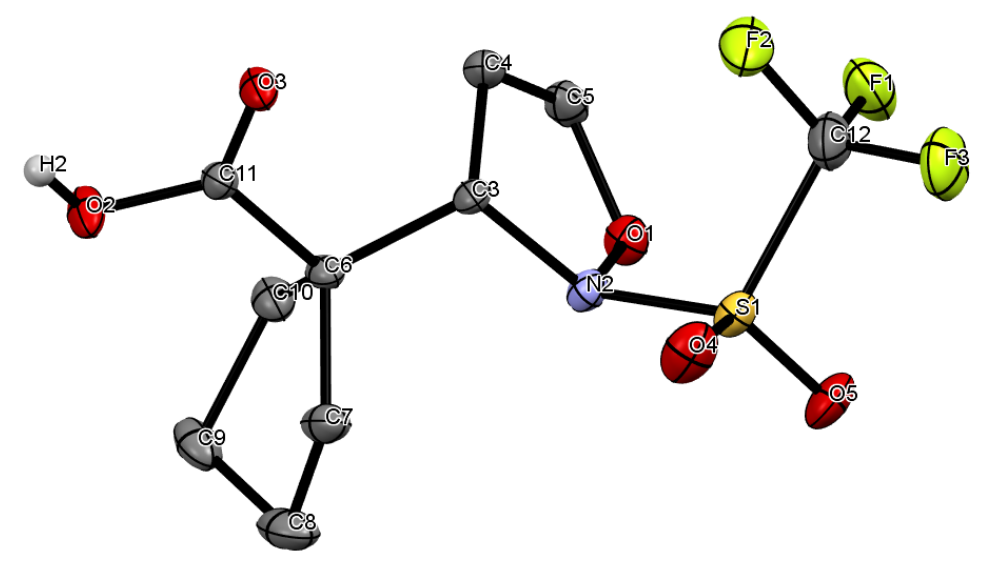

Figure 1. ORTEP view of compound 3c. Thermal ellipsoids at the $30 \%$ probability level.

Interestingly, when the isoxazole is substituted at the 5-position by a methyl group (Scheme 2), the lactonization product 4 was isolated as the main product (Table 3, entries 1 and 2 ), and only some trace amounts of carboxylic acid 3' were observed.

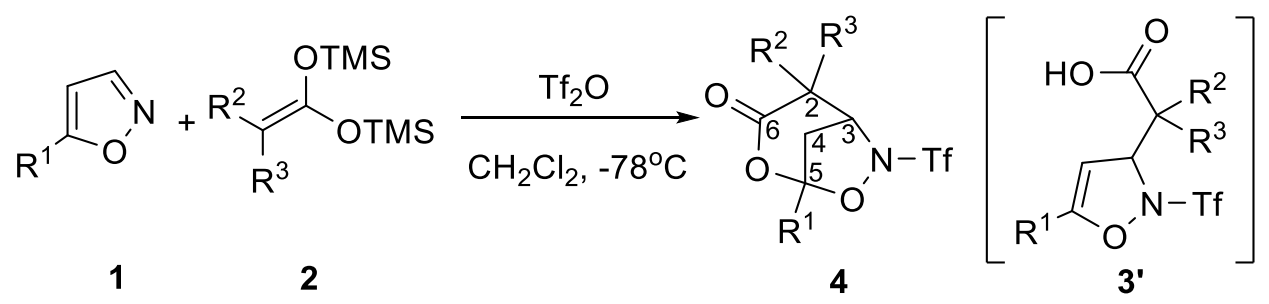

Scheme 2. Synthesis of $\delta$-lactones 4 .

In fact, it was possible to isolate crystals of compound 3' $\left(R^{1}=M e\right.$, entry 2$)$, whose structure was confirmed by X-ray diffraction analysis (Figure 2); however, the amount was not sufficient enough to allow its characterization by NMR spectroscopy. 


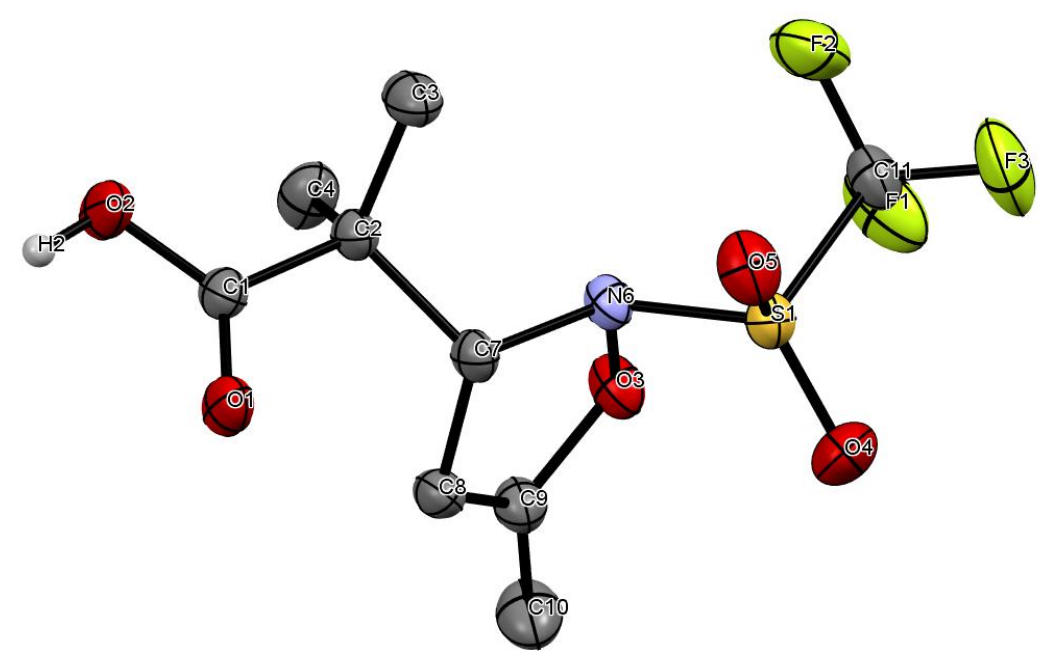

Figure 2. ORTEP view of compound 3'. Thermal ellipsoids at the $30 \%$ probability level.

When the reaction was carried out with phenyl and para-substituted phenyl groups at the 5-position of the isoxazole, the lactonization product 4 (isoxazolidine derivative) was also obtained instead of the carboxylic acid 3. Products 4c-g were obtained in moderate to good yields (Table 3, entries 1-7).

Notably, derivatives $\mathbf{4}$ are easily obtained in only one step using this methodology, while the synthesis reported by Miller and co-workers ${ }^{42}$ for a structurally similar compound required several steps.

Table 3. Yields of isoxazolidine derivates (4)

\begin{tabular}{ccccccc}
\hline Entry & $\mathrm{R}^{1}$ & $\mathrm{R}^{2}$ & $\mathrm{R}^{3}$ & Product & Yield (\%) & Yield 3' \\
\hline 1 & $\mathrm{Me}$ & $\mathrm{Me}^{-\left(\mathrm{CH}_{2}\right)_{5^{-}}}$ & $\mathrm{Ma}$ & 80 & Traces \\
2 & $\mathrm{Me}$ & $\mathrm{Me}$ & $\mathbf{4 b}$ & 40 & Traces \\
3 & $\mathrm{Ph}$ & $\mathrm{Me}$ & $\mathrm{Me}$ & $\mathbf{4 c}$ & 65 & N. O \\
4 & $4-\mathrm{OMePh}$ & $\mathrm{Me}$ & $\mathrm{Me}$ & $\mathbf{4 d}$ & 70 & N. O \\
5 & $4-\mathrm{ClPh}$ & $\mathrm{Me}$ & $\mathrm{Me}$ & $\mathbf{4 e}$ & 50 & N. O \\
6 & $4-\mathrm{FPh}$ & $\mathrm{Me}$ & $\mathrm{Me}$ & $\mathbf{4 f}$ & 54 & N. O \\
7 & $4-\mathrm{BrPh}$ & $\mathrm{Me}$ & $\mathrm{Me}$ & $\mathbf{4 g}$ & 51 & N. O \\
\hline
\end{tabular}

Conditions: Activation time of 12 h, overall reaction time 16 h. N.O (not observed).

The structures of compounds $\mathbf{4 a - g}$ were fully characterized by spectroscopic techniques. As a representative example, for compound $\mathbf{4 e}$, in the IR spectrum, the absorption band of the carbonyl group, $v(C=O)$, was found at $1758 \mathrm{~cm}^{-1}$, which is in the typical range of wavenumbers expected for a $\delta$-lactone. In the ${ }^{1} \mathrm{H}-\mathrm{NMR}$ spectrum, the clearest evidence of lactone formation were protons $\mathrm{H} 3$ and $\mathrm{H} 4$; the signal of $\mathrm{H} 3$ was observed as a doublet at $4.73 \mathrm{ppm}\left({ }^{3} \mathrm{~J}_{\mathrm{H} 3-\mathrm{H} 4} 5.16 \mathrm{~Hz}\right)$, while the signals of the methylene diastereotopic protons $\mathrm{H} 4$ and $\mathrm{H}^{4}$ ' were found at $2.82 \mathrm{ppm}\left(\mathrm{d},{ }^{2} \mathrm{~J}_{\mathrm{H} 4-\mathrm{H} 4^{\prime}} 13.2 \mathrm{~Hz}\right.$ ) and $2.68 \mathrm{ppm}\left(\mathrm{dd}, 2 \mathrm{~J}_{\mathrm{H} 4-\mathrm{H} 4^{\prime}} 13.2 \mathrm{~Hz},{ }^{3} \mathrm{~J}_{\mathrm{H} 3-\mathrm{H} 4} 5.16 \mathrm{~Hz}\right)$, respectively. The ${ }^{13} \mathrm{C}$ spectrum shows the signal of $\mathrm{C} 5$ at 109.9 ppm, corroborating the ring closure, and the quartet signal at $120.9 \mathrm{ppm}\left(J_{\mathrm{CF}}\right.$ $320.25 \mathrm{~Hz}$ ) confirmed the presence of the triflyl group. Furthermore, the molecular structures for lactones $\mathbf{4 c}$ and 4 e were unambiguously confirmed by X-ray diffraction analysis (Figures 3 and 4, respectively). 


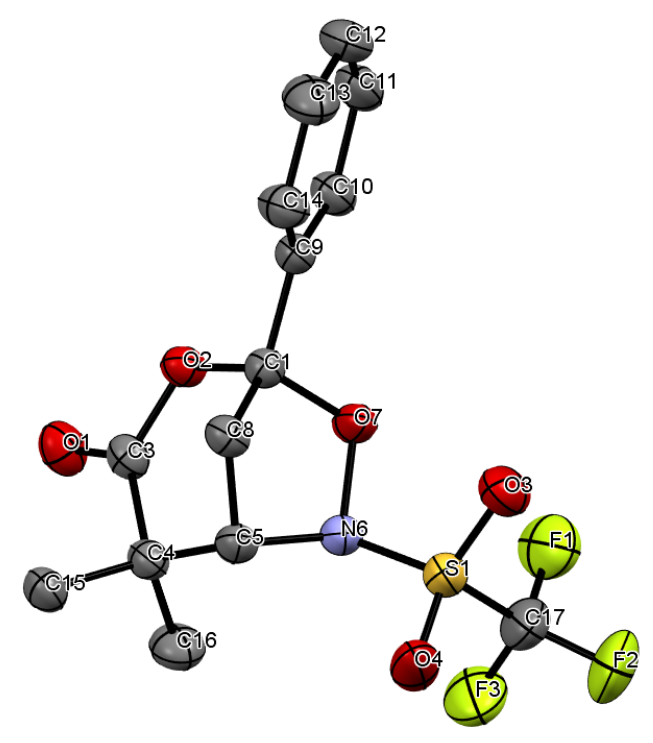

Figure 3. ORTEP view of compound 4c. Thermal ellipsoids at the $30 \%$ probability level.

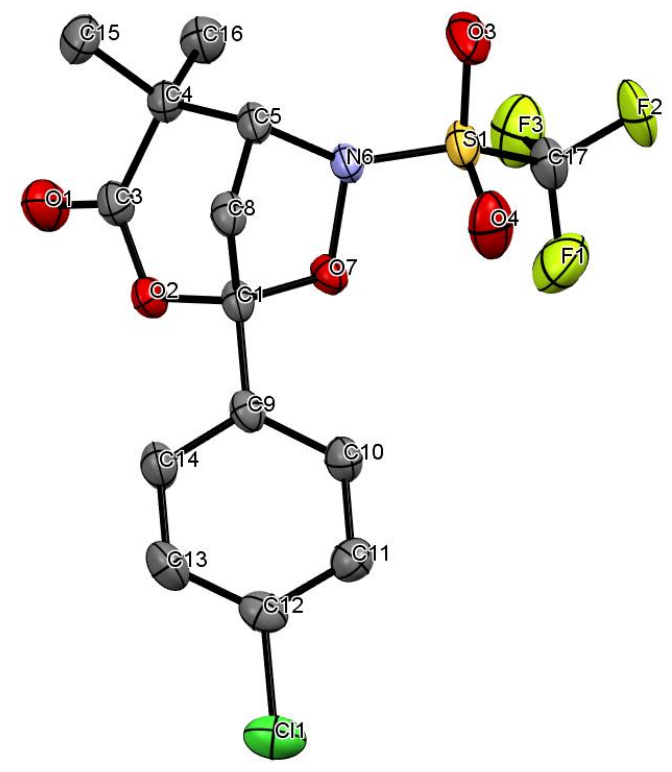

Figure 4. ORTEP view of compound 4e. Thermal ellipsoids at the $30 \%$ probability level.

To explain the regioselectivity of the reaction, a plausible mechanism is proposed as shown in Scheme 3. In the first step, isoxazole 1 reacts with triflic anhydride to form the electrophilic iminium salt $\mathbf{A}$, then ketene acetal $\mathbf{2}$ is activated by triflate anion generating the naked enolate which reacts with iminium salt $\mathbf{A}$ via the nucleophilic addition at imine carbon to yield adduct $\mathbf{B}$. As described above, when isoxazole $\mathbf{1}$ is not substituted at the 5position, the reaction affords the corresponding carboxylic acid $\mathbf{3}$ by hydrolysis of adduct $\mathbf{B}$. In contrast, when the isoxazole is substituted at the 5-position by an electron-donating group like methyl or phenyl, the olefin in adduct $\mathbf{B}$ readily undergoes electrophilic addition, promoted by traces of the triflic acid generated in situ, to give the stabilized carbocation C. Finally, lactone $\mathbf{4}$ is formed through an intramolecular cyclization of intermediate C. In accordance with the stabilization of carbocation $\mathbf{C}$, when the phenyl is substituted at the 4-position by an 
electron-donating group, e.g., a methoxy group (Table 3, entry 2), the lactonization product $\mathbf{4 d}$ was obtained in good yield (70\%), while, with less electron donating groups, e.g., halogens, the yield decreases to around $50 \%$ (Table 3, entries 3-5).

A variety of methodologies have been reported for the olefinic esters cyclization mediated by Bronsted or Lewis acids, all proposed mechanisms for this kind of reaction involve the formation of carbocationic species by addition of the Bronsted or Lewis acid to the olefin, followed by the intramolecular addition of the nucleophile ${ }^{43}$ as shown for transformation B to 4. Although this reaction has been less studied with 2,3-dihidro isoxazol derivatives, a similar reactivity of intermediate $C^{\prime}$ has been proposed by Campagne et al. ${ }^{44}$
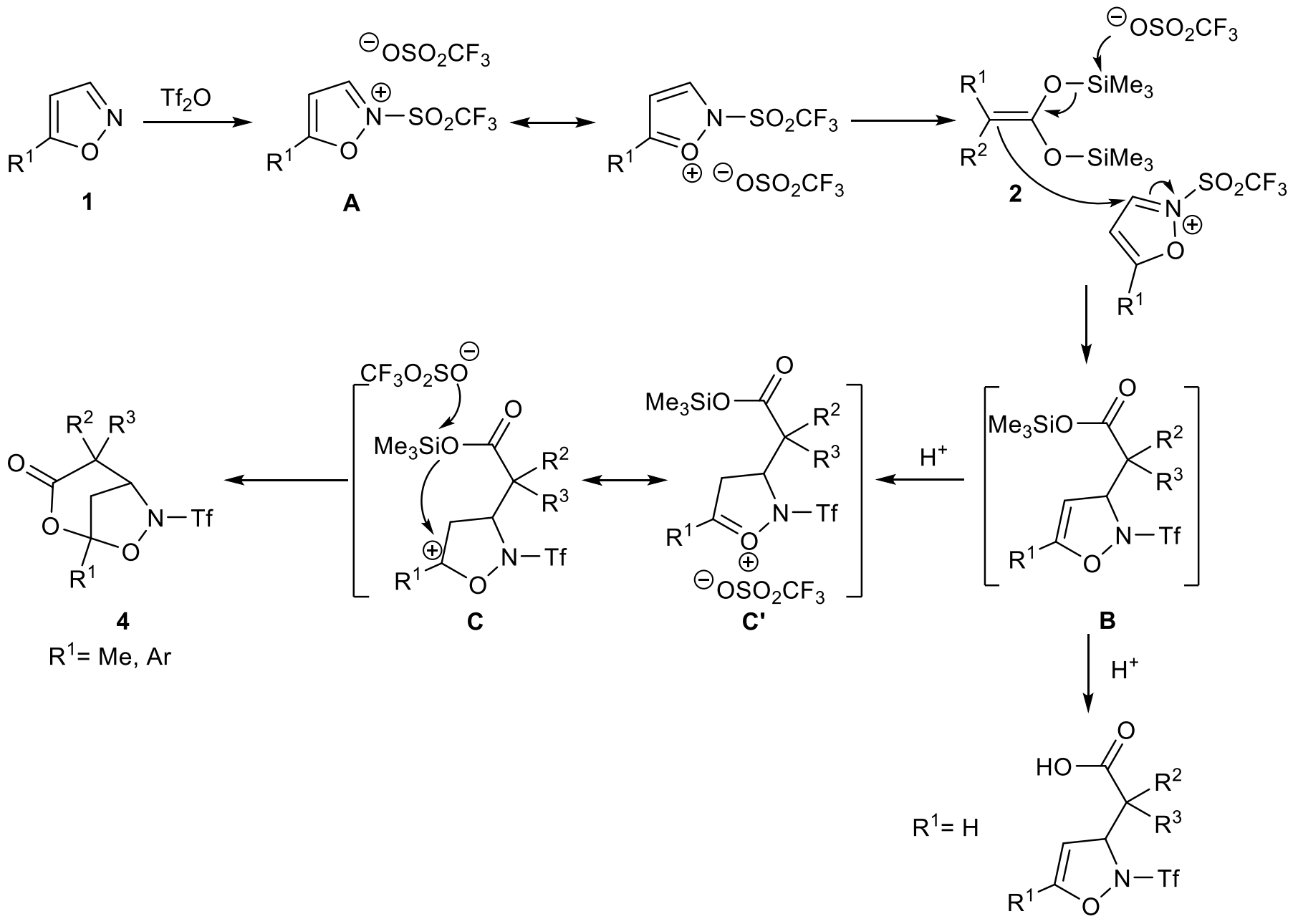

3

Scheme 3. Proposed mechanism for the formation of carboxylic acid $\mathbf{3}$ and $\delta$-lactone $\mathbf{4}$.

\section{Conclusions}

A straightforward methodology has been developed for the synthesis of highly functionalized 4-isoxazoline and isoxazolidine derivatives, via the addition of the masked dinucleophile bis(trimethylsilyl)ketene acetal to $\mathrm{N}$-trifyl activated isoxazoles. The regioselectivity of the reaction is governed by the ability of the substrate to stabilize the carbocation intermediate at the 5-position of the isoxazole. In this way, when the isoxazole is substituted 
by methyl or phenyl groups, the lactonization product (isoxazolidine derivative) is afforded. When the isoxazole is not substituted, the reaction stops at carboxylic acid formation (4-isoxazoline derivative).

\section{Experimental Section}

General. All substrates and solvents were purchased from specialized suppliers with analytical purity and were used as received without any further purification. Melting points were determined on a Melt Temp II apparatus. The ${ }^{1} \mathrm{H}$ and ${ }^{13} \mathrm{C}$ NMR spectra were measured with a Bruker Avance III (300 MHz) and a Bruker Avance III (400 $\mathrm{MHz}$ ) using $\mathrm{CDCl}_{3}$ or Acetone- $d_{6}$ as solvent. Chemical shifts are expressed in ppm $(\delta)$ relative to TMS. The following abbreviations are used: $b r=b r o a d$ signal, $s=$ singlet, $d=d o u b l e t, t=$ triplet, $d d=d o u b l e ~ d o u b l e t, ~ q=q u a r t e t$ and $m=$ multiplet. Mass spectra were obtained by DART and TOF mass spectrometer. IR spectra were obtained with a Bruker TENSOR 27 spectrophotometer. Suitable X-ray-quality crystals of 3c, 3', 4c, 4e compounds were grown through slow evaporation of solvent. A single white crystal of compounds $\mathbf{3 c}, \mathbf{3}^{\prime}, \mathbf{4 c}$, $\mathbf{4 e}$ were mounted on a glass fiber at room temperature. The crystal was then placed on a Bruker SMART APEX CCD diffractometer, equipped with MoKa radiation; decay was negligible all cases. Systematic absences and intensity statistics were used in space group determinations. The structures were determined using direct methods. ${ }^{43}$ Anisotropic structure refinements were achieved using full-matrix, least-squares techniques on all non-hydrogen atoms. All hydrogen atoms were placed in idealized positions, based on hybridization, with isotropic thermal parameters fixed at 1.2 times the value of the attached atom. Structure solutions and refinements were performed using SHELXTL v 6.10. ${ }^{44}$ The bis-(trimethylsilyl)ketene acetals 2 were prepared according to published methods. ${ }^{45}$

General procedure for the synthesis of carboxylic acids 3. Trifluoromethane sulfonic anhydride (1.2 equiv, 3.6 $\mathrm{mmol}$ ) was slowly added by syringe to a solution of isoxazole 1 ( $3 \mathrm{mmol}$ ) in dry dichloromethane $(15 \mathrm{~mL})$ at -78 ${ }^{\circ} \mathrm{C}$ and under an inert atmosphere $\left(\mathrm{N}_{2}\right)$. After $4 \mathrm{~h}$ at $-78{ }^{\circ} \mathrm{C}$, the corresponding bis(trimethylsilyl)ketene acetal 2 ( 1.2 equiv, $3.6 \mathrm{mmol}$ ) was added. Then the temperature was allowed to warm to room temperature and stirred for $20 \mathrm{~h}$. The crude was transferred to a separating funnel and washed with water $(3 \times 20 \mathrm{~mL})$. The organic phase was dried over anhydrous sodium sulfate and the solvent was removed under reduced pressure. The products were purified by silica gel column chromatography, eluted with mixtures of 90:10 hexane/ethyl acetate.

2-Methyl-2-(2-(((trifluoromethyl)sulfonyl)oxy)-2,3-dihydroisoxazol-3-yl)propanoic acid (3a). White powder (1000 mg, 81\%), m.p. 108-110 ${ }^{\circ} \mathrm{C} .{ }^{1} \mathrm{H}$ NMR (300 MHz, CDCl, $\left.\mathrm{ppm}\right): \delta(\mathrm{s}, 1 \mathrm{H},-\mathrm{COOH}), 6.70(\mathrm{dd}, J 1.56,3.06 \mathrm{~Hz}$, $1 \mathrm{H}, \mathrm{H}-2), 5.31(\mathrm{br}, 1 \mathrm{H}, \mathrm{H}-1), 5.23(\mathrm{t}, J 1.47,3.00 \mathrm{~Hz}, 1 \mathrm{H}, \mathrm{H}-3), 1.27\left(\mathrm{~s}, 3 \mathrm{H},-\mathrm{CH}_{3}\right) 1.12\left(\mathrm{~s}, 3 \mathrm{H},-\mathrm{CH}_{3}{ }^{\prime}\right) .{ }^{13} \mathrm{C} \mathrm{NMR}(75$ $\mathrm{MHz}_{2} \mathrm{CDCl}_{3}, \mathrm{ppm}$ ): $\delta 131.38$ (C-5), 144.65 (C-1), 121.74 (q, J $320 \mathrm{~Hz},-\mathrm{CF}_{3}$ ), 100.25 (C-2), 70.41 (C-3), 46.86 (C-4), $22.53\left(\mathrm{CH}_{3}\right), 18.07\left(\mathrm{CH}_{3}{ }^{\prime}\right) . \mathrm{IR}\left(\mathrm{KBr}, \mathrm{cm}^{-1}\right) \vee 3131.31(-\mathrm{OH}), 1771.71(\mathrm{C}=\mathrm{O}) . \mathrm{MS}(\mathrm{DART}, \mathrm{m} / \mathrm{z}): 202\left[\mathrm{M}-\mathrm{C}(\mathrm{CH})_{2} \mathrm{COOH}\right]^{+}$, $290[\mathrm{M}+1]^{+}, 307\left[\mathrm{M}+\mathrm{H}_{2} \mathrm{O}\right]^{+}$. HRMS (DART): calculated for $\mathrm{C}_{8} \mathrm{H}_{11} \mathrm{~F}_{3} \mathrm{NO}_{5} \mathrm{~S}[\mathrm{M}+1]^{+} 290.0200$; found 290.0202.

1-(2-(((Trifluoromethyl)sulfonyl)oxy)-2,3-dihydroisoxazol-3-yl)cyclobutanecarboxylic acid (3b). White powder (537 mg, 41\%), m.p. $116-118^{\circ} \mathrm{C} .{ }^{1} \mathrm{H}$ NMR (400 MHz, Acetone- $\left.d_{6}, \mathrm{ppm}\right): \delta 8.93(\mathrm{~s}, 1 \mathrm{H},-\mathrm{COOH}), 7.21$ (br, J $1.48 \mathrm{~Hz}$, $1 \mathrm{H}, \mathrm{H}-1), 5.48(\mathrm{~m}, 2 \mathrm{H}, \mathrm{H}-2, \mathrm{H}-3), 2.38(\mathrm{~m}, 3 \mathrm{H}, \mathrm{H}-6), 2.01(\mathrm{~m}, 2 \mathrm{H}, \mathrm{H}-7) .{ }^{13} \mathrm{C}$ NMR (100 MHz, Acetone d-6, ppm): $\delta$ 174.19 (C-5), 145.05 (C-1), 121.42 (q, J 300 Hz, -CF $), 100.33$ (C-2), 69.23 (C-3), 50.53 (C-4), 26.63 (C-6), 24.07 (C$\left.6^{\prime}\right), 14.98(\mathrm{C}-7) . \mathrm{IR}\left(\mathrm{KBr}, \mathrm{cm}^{-1}\right) \vee 3130.13(-\mathrm{OH}), 1711.20(\mathrm{C}=\mathrm{O}) . \mathrm{MS}(\mathrm{DART}, \mathrm{m} / \mathrm{z}): 202\left[\mathrm{M}-\mathrm{C}\left(\mathrm{CH}_{3}\right)_{3} \mathrm{COOH}\right]^{+}, 302$ $[\mathrm{M}+1]^{+}, 319\left[\mathrm{M}+\mathrm{H}_{2} \mathrm{O}\right]^{+}$. HRMS (DART): calculated for $\mathrm{C}_{9} \mathrm{H}_{13} \mathrm{~F}_{3} \mathrm{NO}_{5} \mathrm{~S}[\mathrm{M}+1]^{+} 302.0293$; found 302.0283 .

1-(2-(((Trifluoromethyl)sulfonyl)oxy)-2,3-dihydroisoxazol-3-yl)cyclopentanecarboxylic acid (3c). White powder (521 mg, 38\%), m.p. 110-112 ${ }^{\circ} \mathrm{C} .{ }^{1} \mathrm{H}$ NMR (400 MHz, Acetone- $\left.d_{6}, \mathrm{ppm}\right): \delta 7.00$ (dd, $1 \mathrm{H}, J 2.56,4.68 \mathrm{~Hz}$, $\mathrm{H}-1)$, 6.05. (d, J $4.64 \mathrm{~Hz}, 1 \mathrm{H}, \mathrm{H}-2$ ), 5.93 (br, 1H, H-3), $2.18(\mathrm{~m}, 1 \mathrm{H}, \mathrm{H}-6,7), 2.07(\mathrm{~m}, 1 \mathrm{H}, \mathrm{H}-6,7), 1.78(\mathrm{~m}, 6 \mathrm{H}, \mathrm{H}-6,7)$. 
${ }^{13} \mathrm{C}$ NMR (100 MHz, Acetoone- $\left.d_{6}, \mathrm{ppm}\right): \delta 175.34$ (C-5), 126.44 (C-1), 121.46 (q, J $\left.327 \mathrm{~Hz},-\mathrm{CF}_{3}\right), 116.41$ (C-2), 93.00 (C-3), 56.61 (C-4), 32.24 (C-6), 31.89 (C-6') 26.00 (C-7), 25.89 (C-7'). IR (KBr, cm ${ }^{-1}$ ) v $2973.70(-0 H), 1705.97$ $(\mathrm{C}=\mathrm{O})$. MS (DART, m/z): $202\left[\mathrm{M}-\mathrm{C}\left(\mathrm{CH}_{3}\right)_{4} \mathrm{COOH}\right]^{+}, 316[\mathrm{M}+1]^{+}, 333\left[\mathrm{M}+\mathrm{H}_{2} \mathrm{O}\right]^{+}$. HRMS (DART): calculated for $\mathrm{C}_{10} \mathrm{H}_{13} \mathrm{~F}_{3} \mathrm{NO}_{5} \mathrm{~S}[\mathrm{M}+1]^{+} 316.2703$; found 316.2736.

1-(2-(((Trifluoromethyl)sulfonyl)oxy)-2,3-dihydroisoxazol-3-yl)cyclohexanecarboxylic acid (3d). White powder (143 mg, 10\%), m.p. $118-122{ }^{\circ} \mathrm{C} .{ }^{1} \mathrm{H}$ NMR (400 MHz, Acetone-d, $\left.\mathrm{ppm}\right): \delta 7.17$ (dd, J 2.4, $\left.5.0 \mathrm{~Hz}, 1 \mathrm{H}, \mathrm{H}-1\right), 5.50$ (br, J 1.12, $2.6 \mathrm{~Hz}, 1 \mathrm{H}, \mathrm{H}-2), 5.24(\mathrm{br}, 1 \mathrm{H}, \mathrm{H}-3), 2.10$ (m, 3H, H-7, 8, 9), $1.71(\mathrm{~m}, 3 \mathrm{H}, \mathrm{H}-7,8,9), 1.40$ (m, 6H, H-7, 8,9). ${ }^{13} \mathrm{C}$ NMR (100 MHz, Acetone- $\left.d_{6}, \mathrm{ppm}\right): \delta 173.07$ (C-5), 144.98 (C-1), 121.36 (q, J $\left.324 \mathrm{~Hz},-\mathrm{CF}_{3}\right), 99.68$ (C-2), 72.06 (C-3), 51.66 (C-4), 30.01 (C-7), 28.08 (C-7'), 25.24 (C-9), 22.83 (C8), 22.72 (C-8'). IR (KBr, cm $\left.{ }^{-1}\right) \vee 3433.29$ ($\mathrm{OH}), 1701.84(\mathrm{C}=\mathrm{O})$. MS (DART, $\mathrm{m} / z): 202\left[\mathrm{M}-\mathrm{C}\left(\mathrm{CH}_{3}\right)_{5} \mathrm{COOH}\right]^{+}, 330[\mathrm{M}+1]^{+}, 307\left[\mathrm{M}+\mathrm{H}_{2} \mathrm{O}\right]^{+}$. HRMS (DART): calculated for $\mathrm{C}_{11} \mathrm{H}_{14} \mathrm{~F}_{3} \mathrm{NO}_{5} \mathrm{~S}[\mathrm{M}+1]^{+} 330.2921$; found 330.2912 .

General procedure for the synthesis of lactones 4. Trifluoromethane sulfonic anhydride (1.2 equiv, $3.6 \mathrm{mmol})$ was slowly added by syringe to a solution of aryl isoxazole $1(3 \mathrm{mmol})$ in dry dichloromethane $(15 \mathrm{~mL})$ at $-78^{\circ} \mathrm{C}$ and under an inert atmosphere $\left(\mathrm{N}_{2}\right)$. After $12 \mathrm{~h}$ at $-78{ }^{\circ} \mathrm{C}$, the corresponding bis(trimethylsilyl)ketene acetal 2 (1.2 equiv, $3.6 \mathrm{mmol}$ ) was added. Then the temperature was allowed to warm to room temperature and stirred for $4 \mathrm{~h}$. The crude mixture was transferred to a separating funnel and washed with water ( $3 \times 20 \mathrm{~mL})$. The organic phase was dried over anhydrous sodium sulfate and the solvent was removed under reduced pressure. The products were purified by silica gel column chromatography, eluted with mixtures of 90:10 hexane/ethyl acetate.

1-Methyl-3-oxo-2,7-dioxa-6-azaspiro[bicyclo[3.2.1]octane-4,1'-cyclohexan]-6-yl trifluoromethanesulfonate (4a). White powder (1000 mg, 80\%), m.p. $78-80{ }^{\circ} \mathrm{C} .{ }^{1} \mathrm{H}$ NMR $\left(400 \mathrm{MHz}\right.$, Acetone- $\left.d_{6}, \mathrm{ppm}\right): \delta 5.23(\mathrm{~d}, J 5.52 \mathrm{~Hz}$, $1 \mathrm{H}, \mathrm{H}-1), 3.04$ (d, J 13.6, 1H, H-3) 2.55 (dd, J 5.52, 13.56, 1H, H-2), $1.92(\mathrm{~m}, 4 \mathrm{H}, \mathrm{H}-7,8,9), 1.79(\mathrm{~s}, 3 \mathrm{H}, \mathrm{H}-6), 1.47$ (m. 6H, H-7, 8, 9). ${ }^{13} \mathrm{C}$ NMR (100 MHz, Acetone- $\left.d_{6}, \mathrm{ppm}\right): \delta 171.85$ (C-5), 120.66 (q, J $\left.321 \mathrm{~Hz},-\mathrm{CF}_{3}\right), 109.47$ (C-1), 61.29 (C-2), 48.04 (C-3), 38.51 (C-4), 31.95 (C-7), 31.13 (C-7'), 24.77 (C-6), 20.52 (C-9), 19.70 (C-8), 19.18 (C-8'). $\mathrm{IR}\left(\mathrm{KBr}, \mathrm{cm}^{-1}\right) \vee 1734.86(\mathrm{C}=\mathrm{O}) . \mathrm{MS}(\mathrm{DART}, \mathrm{m} / z): 210[\mathrm{M}-\mathrm{Tf}]^{+}, 344[\mathrm{M}+1]^{+}, 361\left[\mathrm{M}+\mathrm{H}_{2} \mathrm{O}\right]^{+}$. HRMS (DART): calculated for $\mathrm{C}_{12} \mathrm{H}_{17} \mathrm{~F}_{3} \mathrm{NO}_{5} \mathrm{~S}[\mathrm{M}+1]^{+}$344.07795; found 344.07738.

1,4,4-Trimethyl-3-oxo-2,7-dioxa-6-azabicyclo[3.2.1]octan-6-yl trifluoromethanesulfonate (4b). White powder (1000 mg, 40\%), m.p. 82-84 ${ }^{\circ} \mathrm{C} .{ }^{1} \mathrm{H}$ NMR (400 MHz, Acetone- $\left.d_{6}, \mathrm{ppm}\right): \delta 4.86$ (d, J $\left.5.32 \mathrm{~Hz}, 1 \mathrm{H}, \mathrm{H}-1\right), 3.12$ (d, J 13.56, $1 \mathrm{H}, \mathrm{H}-3) 2.50$ (dd, J 5.40, $13.60 \mathrm{~Hz}, 1 \mathrm{H}, \mathrm{H}-2), 1.79(\mathrm{~s}, 3 \mathrm{H}, \mathrm{H}-6) 1.47\left(\mathrm{~s}, 3 \mathrm{H},-\mathrm{CH}_{3}\right) 1.41\left(\mathrm{~s}, 3 \mathrm{H},-\mathrm{CH}_{3}{ }^{\prime}\right) .{ }^{13} \mathrm{C} \mathrm{NMR}$ (100 MHz, Acetone- $\left.d_{6}, \mathrm{ppm}\right): \delta 172.30$ (C-5), 120.77 (q, J $321 \mathrm{~Hz},-\mathrm{CF}_{3}$ ), 109.88 (C-1), 65.74 (C-2), 44.50 (C-4) 38.51 (C-3) $24.53\left(-\mathrm{CH}_{3}\right), 23.16\left(-\mathrm{CH}_{3}\right), 19.16(\mathrm{C}-6)$. IR $\left(\mathrm{KBr}, \mathrm{cm}^{-1}\right) \vee 1742.91$ (C=O). MS (DART, m/z): 170 [M-Tf] , $304[\mathrm{M}+1]^{+}, 321\left[\mathrm{M}+\mathrm{H}_{2} \mathrm{O}\right]^{+}$. HRMS (DART): calculated for $\mathrm{C}_{9} \mathrm{H}_{13} \mathrm{~F}_{3} \mathrm{NO}_{5} \mathrm{~S}[\mathrm{M}+1]^{+} 304.04665$; found 304.04796.

4,4-Dimethyl-3-oxo-1-phenyl-2,7-dioxa-6-azabicyclo[3.2.1]octan-6-yl trifluoromethanesulfonate (4c). White powder (490 mg, 65\%), m.p. 88-90 ${ }^{\circ} \mathrm{C} .{ }^{1} \mathrm{H} \mathrm{NMR}\left(300 \mathrm{MHz}, \mathrm{CDCl}_{3}, \mathrm{ppm}\right): \delta 7.67(\mathrm{~m}, J 7.41,3.75 \mathrm{~Hz}, 2 \mathrm{H}, \mathrm{H}-7), 7.42$ $(\mathrm{m}, J$ 5.5, $2.28 \mathrm{~Hz}, 3 \mathrm{H}, \mathrm{H}-8), 4.71(\mathrm{~m}, J 5.07 \mathrm{~Hz}, 1 \mathrm{H}, \mathrm{H}-2), 2.87(\mathrm{~d}, J 13.26 \mathrm{~Hz}, 1 \mathrm{H}, \mathrm{H}-3), 2.67(\mathrm{dd}, J 5.04,13.31 \mathrm{~Hz}$, $\left.1 \mathrm{H}, \mathrm{H}-3^{\prime}\right) 1.49\left(\mathrm{~s}, 3 \mathrm{H},-\mathrm{CH}_{3}\right), 1.42\left(\mathrm{~s}, 3 \mathrm{H},-\mathrm{CH}_{3}{ }^{\prime}\right) .{ }^{13} \mathrm{C} \mathrm{NMR}\left(75 \mathrm{MHz}, \mathrm{CDCl}_{3}, \mathrm{ppm}\right): \delta 172.44$ (C-4), 131.99 (C-6), 130.37 (C-8), 128.77 (C-9), 126.12 (C-7), 116.74 (q, J 320 Hz, -CF $), 110.29$ (C-5), 65.52 (C-2), 44.69 (C-3), 41.29 (C-1), 25.37, $23.60\left(-\mathrm{CH}_{3}\right)$. IR $\left(\mathrm{KBr}, \mathrm{cm}^{-1}\right) 1751.25$ (C=O). MS (DART, m/z): $278\left[\mathrm{M}-\mathrm{C}\left(\mathrm{CH}_{3}\right){ }_{2} \mathrm{COO}\right]^{+}, 366[\mathrm{M}+1]^{+}$, $367[\mathrm{M}+2]^{+}$. HRMS (DART): calculated for $\mathrm{C}_{14} \mathrm{H}_{15} \mathrm{~F}_{3} \mathrm{NO}_{5} \mathrm{~S}[\mathrm{M}+1]^{+} 366.0578$; found 366.0546.

1-(4-Methoxyphenyl)-4,4-dimethyl-3-oxo-2,7-dioxa-6-azabicyclo[3.2.1]octan-6-yl trifluoromethanesulfonate (4d). White powder (472 mg, 70\%), m.p. 82-90 ${ }^{\circ} \mathrm{C} .{ }^{1} \mathrm{H} \mathrm{NMR}\left(300 \mathrm{MHz}, \mathrm{CDCl}_{3}, \mathrm{ppm}\right): \delta 7.61(\mathrm{~d}, J 8.4 \mathrm{~Hz}, 2 \mathrm{H}, \mathrm{H}-7)$, 6.95 (d, J 8.4, 2H, H-8), 4.69 (d, J $5.1 \mathrm{~Hz}, 1 \mathrm{H}, \mathrm{H}-2$ ), 3.81 (s, 3H, H-10), 2.84 (d, J $12.9 \mathrm{~Hz}, 1 \mathrm{H}, \mathrm{H}-3$ ) 2.72 (dd, J 5.1, $\left.13.3 \mathrm{~Hz}, 1 \mathrm{H}, \mathrm{H}-3^{\prime}\right), 1.51\left(\mathrm{~s}, 3 \mathrm{H},-\mathrm{CH}_{3}\right), 1.45$ (s, 3H, $\left.-\mathrm{CH}_{3}{ }^{\prime}\right) .{ }^{13} \mathrm{C} \mathrm{NMR}\left(75 \mathrm{MHz}, \mathrm{CDCl}_{3}, \mathrm{ppm}\right): \delta 172.54$ (C-4), 161.10 (C-9), 127.67 (C-7), 123.69 (C-6), 120.97 (q, J 320 Hz, -CF ), 114.10 (C-8), 110.45 (C-5), 65.47 (C-2), 55.30 (C-10), 
44.66 (C-3), 41.27 (C-1), 25.55, $23.68\left(-\mathrm{CH}_{3}\right) . \mathrm{IR}\left(\mathrm{KBr}, \mathrm{cm}^{-1}\right) \vee 3432.57\left(\mathrm{O}-\mathrm{CH}_{3}\right), 1761.56$ (C=O). MS (DART, $\left.m / z\right)$ : $262[\mathrm{M}-\mathrm{Tf}]^{+}, 396[\mathrm{M}+1]^{+}, 367\left[\mathrm{M}+\mathrm{H}_{2} \mathrm{O}\right]^{+}$. HRMS (DART): calculated for $\mathrm{C}_{15} \mathrm{H}_{17} \mathrm{~F}_{3} \mathrm{NO}_{6} \mathrm{~S}[\mathrm{M}+1]^{+} 396.0729$; found 396.07134.

1-(4-Chlorophenyl)-4,4-dimethyl-3-oxo-2,7-dioxa-6-azabicyclo[3.2.1]octan-6-yl trifluoromethanesulfonate (4e). White powder (332 mg, 50\%), m.p. 98-100 ${ }^{\circ} \mathrm{C} .{ }^{1} \mathrm{H} \mathrm{NMR}\left(300 \mathrm{MHz}, \mathrm{CDCl}_{3}, \mathrm{ppm}\right): \delta 7.66(\mathrm{~d}, J 8.61 \mathrm{~Hz}, 2 \mathrm{H}, \mathrm{H}-$ 7), 7.43 (d, J $8.64 \mathrm{~Hz}, 2 \mathrm{H}, \mathrm{H}-8$ ), 2.87 (d, J $5.1 \mathrm{~Hz}, 2 \mathrm{H}, \mathrm{H}-2), 2.72$ (d, J $13.20 \mathrm{~Hz}, 1 \mathrm{H}, \mathrm{H}-3$ ), 2.68 (dd, J 5.16, 13.23 Hz, $\left.1 \mathrm{H}, \mathrm{H}-3^{\prime}\right) 1.53\left(\mathrm{~s}, 3 \mathrm{H},-\mathrm{CH}_{3}\right), 1.49\left(\mathrm{~s}, 3 \mathrm{H},-\mathrm{CH}_{3}{ }^{\prime}\right) .{ }^{13} \mathrm{C} \mathrm{NMR}\left(75 \mathrm{MHz}, \mathrm{CDCl}_{3}, \mathrm{ppm}\right): \delta 171.12$ (C-4), 136.62 (C-9), 130.48 (C-6), 129.07 (C-7), 127.63 (C-8), 120.88 (q, J 320 Hz, -CF $), 109.80$ (C-5), 65.38 (C-2), 44.72 (C-1), 41.66 (C-3), 25.65, $23.67\left(-\mathrm{CH}_{3}\right)$. IR ( $\left.\mathrm{KBr}, \mathrm{cm}^{-1}\right) \vee 1758.59$ (C=O). MS (DART, m/z): $311\left[\mathrm{M}-\mathrm{C}\left(\mathrm{CH}_{3}\right)_{2} \mathrm{COCl}\right]^{+}, 400(\mathrm{Cl}, 35)$, $402(\mathrm{Cl}, 37)[\mathrm{M}+1]^{+}$. HRMS (DART): calculated for $\mathrm{C}_{14} \mathrm{H}_{14} \mathrm{ClF}_{3} \mathrm{NO}_{5} \mathrm{~S}[\mathrm{M}+1]^{+} 400.0233$; found 400.0237.

1-(4-Fluorophenyl)-4,4-dimethyl-3-oxo-2,7-dioxa-6-azabicyclo[3.2.1]octan-6-yl trifluoromethanesulfonate (4f). White powder (426 mg, 54\%), m.p. $92-94{ }^{\circ} \mathrm{C} .{ }^{1} \mathrm{H} \mathrm{NMR}\left(400 \mathrm{MHz}, \mathrm{CDCl}_{3}, \mathrm{ppm}\right): \delta 7.74(\mathrm{dq}, J 14.01,3.81 \mathrm{~Hz}$, $2 \mathrm{H}, \mathrm{H}-8$ ), 7.19 (t, J 9.15, 8.58 Hz, 2H, H-7), 4.73 (d, J $5.04 \mathrm{~Hz}, 1 \mathrm{H}, \mathrm{H}-3$ ), 2.87 (d, J $13.20 \mathrm{~Hz}, 1 \mathrm{H}, \mathrm{H}-3), 2.75$ (dd, J 13.2, $5.07 \mathrm{~Hz}, 1 \mathrm{H}, \mathrm{H}-3), 1.56\left(\mathrm{~s}, 3 \mathrm{H},-\mathrm{CH}_{3}\right), 1.51\left(\mathrm{~s}, 3 \mathrm{H},-\mathrm{CH}_{3}{ }^{\prime}\right) .{ }^{13} \mathrm{C} \mathrm{NMR}\left(75 \mathrm{MHz}, \mathrm{CDCl}_{3}, \mathrm{ppm}\right): \delta 172.18(\mathrm{C}-4)$, 165.53 (d, 249, C-7) 128.55 (d, 9, C-7), 127.85 (d, 3, C-6), 120.90 (q, J 320 Hz, -CF ), 116.63 (d, 42, C-8), 109.91 (C-5), 65.36 (C-2), 44.71 (C-1), 41.79 (C-3), 25.75, $23.72\left(-\mathrm{CH}_{3}\right) . \mathrm{IR}\left(\mathrm{KBr}, \mathrm{cm}^{-1}\right) \vee 1770.24(\mathrm{C}=\mathrm{O}) . \mathrm{MS}$ (DART, $\left.m / z\right)$ : $339[\mathrm{M}-\mathrm{COOF}]^{+}, 384[\mathrm{M}+1]^{+}$. HRMS (DART): calculated for $\mathrm{C}_{14} \mathrm{H}_{14} \mathrm{~F}_{4} \mathrm{NO}_{5} \mathrm{~S}[\mathrm{M}+1]^{+} 384.0603$; found 384.0603 .

1-(4-Bromophenyl)-4,4-dimethyl-3-oxo-2,7-dioxa-6-azabicyclo[3.2.1]octan-6-yl trifluoromethanesulfonate (4g). White powder (303 mg, 51\%), m.p. 88-90 ${ }^{\circ} \mathrm{C} .{ }^{1} \mathrm{H} \mathrm{NMR}\left(300 \mathrm{MHz}, \mathrm{CDCl}_{3}, \mathrm{ppm}\right): \delta 7.61(\mathrm{~s}, 2 \mathrm{H}, \mathrm{H}-8), 7.28(\mathrm{~s}$, $2 \mathrm{H}, \mathrm{H}-7$ ), 2.87 (d, J $5.1 \mathrm{~Hz}, 2 \mathrm{H}, \mathrm{H}-2$ ), 4.73 (d, J $5.04 \mathrm{~Hz}, 1 \mathrm{H}, \mathrm{H}-2$ ), 2.86 (d, J $13.17 \mathrm{~Hz}, 1 \mathrm{H}, \mathrm{H}-3^{\prime}$ ), 2.74 (dd, J 5.1 , $\left.13.17 \mathrm{~Hz}, 1 \mathrm{H}, \mathrm{H}-3^{\prime}\right), 1.56\left(\mathrm{~s}, 3 \mathrm{H},-\mathrm{CH}_{3}\right), 1.51$ (s, 3H, $\left.-\mathrm{CH}_{3}{ }^{\prime}\right) .{ }^{13} \mathrm{C} \mathrm{NMR}\left(75 \mathrm{MHz}, \mathrm{CDCl}_{3}, \mathrm{ppm}\right): \delta 172.05$ (C-4), 132.08 (C-8), 130.96 (C-9), 127.99 (C-7), 124.89 (C-6), 109.81 (C-5), 65.33 (C-2), 44.74 (C-1), 41.77 (C-3), 25.79, 23.73 ($\left.\mathrm{CH}_{3}\right)$. IR $\left(\mathrm{KBr}, \mathrm{cm}^{-1}\right) \vee 1733.21(\mathrm{C}=0)$.MS (DART, m/z): $355(\mathrm{Br}, 79), 357(\mathrm{Br}, 81)\left[\mathrm{M}-\mathrm{CF}_{3} \mathrm{~S}\right]^{+}, 443(\mathrm{Br}, 79), 445(\mathrm{Br}$, 81) $[\mathrm{M}+1]^{+}$. HRMS (DART): calculated for $\mathrm{C}_{14} \mathrm{H}_{14} \mathrm{BrF}_{3} \mathrm{NO}_{5} \mathrm{~S}[\mathrm{M}+1]^{+} 443.9729$; found 443.9743.

\section{Acknowledgements}

This work was financially supported by DGAPA PAPIIT Project IN203120. We thank Maria del Rocio Patiño Maya and María del Carmen García González for their technical assistance.

\section{Supplementary Material}

Copies of ${ }^{1} \mathrm{H}$ and ${ }^{13} \mathrm{C}$ NMR spectra associated with this manuscript are presented in the Supplementary Material file in the online version.

\section{References}

1. Kaur, K.; Kumar, V.; Sharma, A. K.; Gupta, G. K. Eur. J. Med. Chem. 2014, 77, 121-133. https://doi.org/10.1016/i.ejmech.2014.02.063

2. Wang, Y.; Shao, Y.; Wang, Y.; Fan, L.; Yu, X.; Zhi, X.; Yang, C.; Qu, H.; Yao, X.; Xu, H. J. Agric. Food Chem. 2012, $60,8435-8443$. 
https://doi.org/10.1021/jf303069v

3. Yang, R.; Zhang, Y.; Xu, H. Bioorg. Med. Chem. Lett. 2018, 28, 1410-1416.

https://doi.org/10.1016/i.bmcl.2018.02.018

4. Kalaria, P. N.; Satasia, S. P.; Raval, D. K. New J. Chem. 2014, 38, 2902-2910.

https://doi.org/10.1039/C4NJ00244J

5. Nikam, M. D.; Mahajan, P. S.; Damale, M. G.; Sangshetti, J.N.; Dabhade, S.K.; Shinde, D. W.; Gill, C. H. Med. Chem. Res. 2015, 24, 3372-3386.

https://doi.org/10.1007/s00044-015-1385-x

6. Miglianico, M.; Eldering, M.; Slater, H.; Ferguson, N.; Ambrose, P.; Lees, R. S.; Koolen, K. M. J.; Pruzinova, K.; Jancarova, M.; Volf, P.; Koenraadt, C. J. M.; Duerr, H. P.; Trevitt, G.; Yang, B.; Chatterjee, A. K.; Wisler, J.; Sturm, A.; Bousema, T.; Sauerwein, R. W.; Schultz, P. G.; Tremblay, M. S.; Dechering, K. J. PNAS 2018, 115, E6920-E6926.

https://dx.doi.org/10.1073/pnas.1801338115

7. Berthet, M.; Cheviet, T.; Dujardin, G.; Parrot, I.; Martinez, J. Chem. Rev. 2016, 116, 15235-15283.

https://doi.org/10.1021/acs.chemrev.6b00543

8. Maslat, A. O.; Bkhaitan, M.; Sheikha, G. A. Drug Chem. Toxicol. 2007, 30, 41-53. https://doi.org/10.1080/01480540601017652

9. Mosbah, H.; Chahdoura, H.; Mannai, A.; Snoussi, M.; Aouadi, K.; Abreu, R. M. V.; Bouslama, A.; Achour, L.; Selmi, B. Applied Biochemistry and Biotechnology 2019, 187, 1113-1130. https://doi.org/10.1007/s12010-018-2868-2

10. Nge, C. E.; Sim, K. S.; Lim, S. H.; Thomas, N. F.; Low, Y. Y.; Kam, T. S.; J. Nat. Prod. 2016, 79, $2709-2717$. https://doi.org/10.1021/acs.jnatprod.6b00674

11. Yamaguchi, M.; Matsuda, A.; Ichikawa, S. Org. Biomol. Chem. 2015, 13, 1187-1197. https://doi.org/10.1039/C4OB02142H

12. Das, P.; Hamme, A. T. Eur. J.Org. Chem. 2015, 2015, 5159-5166. https://doi.org/10.1002/ejoc.201500603

13. Kozikowski, A. P. Acc. Chem. Res. 1984, 17, 410-416. https://doi.org/10.1021/ar00108a001

14. Choe, H.; Pham, T. T.; Lee, J. Y.; Latif, M.; Park, H.; Kang, Y. K.; Lee, J. J. Org. Chem. 2016, 81, $2612-2617$. https://doi.org/10.1021/acs.joc.5b02760

15. Diethelm, S.; Carreira, E. M. J. Am. Chem. Soc. 2013, 135, 8500-8503. https://doi.org/10.1021/ja403823n

16. Diethelm, S.; Carreira, E. M. J. Am. Chem. Soc. 2015, 137, 6084-6096. https://doi.org/10.1021/jacs.5b02574

17. Hubert, J. G.; Stepek, I. A.; Noda, H.; Bode, J. W. Chem. Sci. 2018, 9, 2159-2167. https://doi.org/10.1039/C7SC05057G

18. Singh, G.; Raj. T.; Gupta, V.; Ishar, M. P. S. Tetrahedron Lett. 2016, 57, 4688-4692. https://doi.org/10.1016/i.tetlet.2016.09.011

19. Wang, X. J.; Zhang, G. J.; Zhuang, P. Y.; Zhang, Y.; Yu, S. S.; Bao, X. Q.; Zhang, D.; Yuan, Y. H.; Chen, N. H.; Ma, S. G.; Qu, J.; Li, Y. Org. Lett. 2012, 14, 2614-2617. https://doi.org/10.1021/ol3009478

20. Zhang, G. L.; Rücker, G.; Breitmaier, E.; Nieger. M.; Mayer, R.; Steinbeck, C. Phytochemistry 1995, 40, 299305. 
21. Tsuda, M.; Hirano, K.; Kubota, T.; Kobayashi, J. Tetrahedron Lett. 1999, 40, 4819-4820. https://doi.org/10.1016/S0040-4039(99)00852-7

22. Álvarez-Toledano, C.; Penieres-Carrillo, J. G. Eur. J. Org. Chem. 2018, 2018, 4040-4049. https://doi.org/10.1002/ejoc.201800227

23. Rivera-Hernández, A.; Chans, G.;M.; Rudler, H.; López-Cortés, J. G.; Toscano, R. A.; Álvarez-Toledano C. Tetrahedron 2014, 70, 1861-1871.

https://doi.org/10.1016/j.tet.2014.01.044

24. Ballinas-Indili, R.; Corona-Sánchez, R.; Merecías, R. S.; M. Teresa Ramírez-Apan, M. T.; Toscano, A.; LomasRomero, L.; Guerrero-Reyes, R.; Álvarez-Toledano, C. Arkivoc, 2019, (v), 327-339.

https://doi.org/10.24820/ark.5550190.p010.876

25. Parlier, A.; Kadouri-Puchot, C.; Beaupierre, S.; Jarosz, N.; Rudler, H.; Hamon, L.; Herson, P.; Daran, J. C. Tetrahedron Lett. 2009, 50, 7274-7279.

https://doi.org/10.1016/i.tetlet.2009.10.038

26. Rudler, H.; Denise, B.; Parlier, A.; Daran, J. C. Chem. Commun. 2002, 2, 940-941. https://doi.org/10.1039/B201780F

27. Xu, Y.; Aldeco-Pérez, E.; Rudler, H.; Parlier, A.; Alvarez, C. Tetrahedron Lett. 2006, 47, 4553-4556. https://doi.org/10.1016/j.tetlet.2006.05.006

28. Rudler, H.; Parlier, A.; Sandoval-Chavez, C.; Herson, P.; Daran, J. C. Angew. Chem. Int. Ed. 2008; 47, 68436846.

https://doi.org/10.1002/anie.200801879

29. Rudler, H.; Denise, B.; Xu, Y.; Parlier, A.; Vaissermann, J. Eur. J. Org. Chem. 2005, 3724-3744. https://doi.org/10.1002/ejoc.200500162

30. Garduno-Alva, A.; Xu, Y.; Gualo-Soberanes, N.; Lopez-Cortes, J.; Rudler, H.; Parlier, A.; Ortega-Alfaro, M. C.; Alvarez-Toledano, C.; Toscano, R. A. Eur. J. Org. Chem. 2008, 3714-3723.

https://doi.org/10.1002/ejoc.200800338

31. Garduño-Alva, A.; Ortega-Alfaro, M. C.; López-Cortés, J.; G.; Chávez, I.; Barroso-Flores, J.; Toscano, R. A.; Rudler, H.; Álvarez-Toledano, C. Can. J. Chem. 2012, 90, 469-482.

https://doi.org/10.1139/v2012-016

32. Schmidt, A.; Michalik, D.; Rotzoll, S.; Ullah, E.; Fischer, C.; Reinke, H.; Görls, H.; Langer, P. Org. Biomol. Chem. 2008, 6, 2804-2814.

https://doi.org/10.1039/B804139C

33. Ullah, E.; Rotzoll, S.; Schmidt. A.; Michalik, D.; Langer, P. Tetrahedron Lett. 2005, 46, 8997-8999. https://doi.org/10.1016/j.tetlet.2005.10.108

34. Rivera-Hernández, A.; López-Jimeno, I. S.; Carmona-Reyes, G. A.; Alfredo-Toscano, R.; Penieres-Carrillo, J. G.; Álvarez-Toledano, C. Tetrahedron Lett. 2015, 56, 4829-4832.

https://doi.org/10.1016/i.tetlet.2015.06.069

35. Valencia-Galicia, N. A.; Corona-Sánchez, R.; Ballinas-Indili, R.; Toscano, R. A.; Macías-Rubalcava, M. L.; Álvarez-Toledano, C. Tetrahedron Lett. 2017, 58, 3168-3171.

https://doi.org/10.1016/j.tetlet.2016.10.013

36. Gómez-García, O.; Gómez, E.; Toscano, R. A.; Salgado-Zamora, H.; Álvarez-Toledano C. Synthesis 2016, 48, 1371-1380.

https://doi.org/10.1055/s-0035-1560422

37. Lavilla, R.; Spada, A.; Bosch. J. J. Org. Chem. 2001, 66, 1487-1491.

https://doi.org/10.1021/jo001407p 
38. Lavilla, R. J. Chem. Soc., Perkin Trans. 1 2002, 2, 1141-1156.

https://doi.org/10.1039/B101371H

39. Wenkert, E.; Chang, C.; J.; Chawla, H. P. S.; Cochran, D. W.; Hagaman, E.; W.; King, J. C.; Orito, K. J. Am. Chem. Soc. 1976, 98, 3645-3655.

https://doi.org/10.1021/ja00428a044

40. Kawai, H.; Sugita, Y.; Tokunaga, E.; Sato, H.; Shiro, M.; Shibata, N. ChemistryOpen 2014, 3, $14-18$. https://doi.org/10.1002/open.201300044

41. Xu, X. H.; Matsuzaki, K.; Shibata, N. Chem. Rev. 2015, 115, 731-764.

https://doi.org/10.1021/cr500193b

42. Williams, M. A.; Miller, M. J.; Rath, N. P. J. Org. Chem. 1991, 56, 1293-1296.

https://doi.org/10.1021/jo00003a066

43. Muñoz, M. P.; Lloyd-Jones, G. C. Eur. J. Org. Chem. 2009, 516-524.

https://doi.org/10.1002/ejoc.200800970

44. Debleds, O.; Zotto, C. D.; Vrancken, E.; Campagne, J. M.; Retailleau, P. Adv. Synth. Catal. 2009, 351,1991 1998

https://doi.org/10.1002/adsc.200900127

45. Wissner, A. J. Org. Chem. 1979, 44, 4617-4622

https://doi.org/10.1021/jo00393a034

46. Altomare, A.; Cascarano, G.; Giacovazzo, C.; Guagliardi, A.; Burla, M. C.; Polidori, G.; Canalli, M. J. Appl. Crystallogr. 1994, 27, 435-435.

https://doi.org/10.1107/S002188989400021X

47. Sheldrick, G. M. Acta Crystallogr. Sect. A 2008, 64, 112-122.

https://doi.org/10.1107/S0108767307043930

48. Ainsworth, C.; Kuo, Y. N. J. Organomet. Chem. 1972, 46, 73-87.

https://doi.org/10.1016/S0022-328X(00)90476-5 